\title{
Myoelectric control of prosthetic hands: state-of-the-art review
}

This article was published in the following Dove Press journal:

Medical Devices: Evidence and Research

27 July 2016

Number of times this article has been viewed

\section{Purushothaman Geethanjali \\ School of Electrical Engineering Department of Control and Automation VIT University, Vellore, Tamil Nadu, India}

\begin{abstract}
Myoelectric signals (MES) have been used in various applications, in particular, for identification of user intention to potentially control assistive devices for amputees, orthotic devices, and exoskeleton in order to augment capability of the user. MES are also used to estimate force and, hence, torque to actuate the assistive device. The application of MES is not limited to assistive devices, and they also find potential applications in teleoperation of robots, haptic devices, virtual reality, and so on. The myoelectric control-based prosthetic hand aids to restore activities of daily living of amputees in order to improve the self-esteem of the user. All myoelectric control-based prosthetic hands may not have similar operations and exhibit variation in sensing input, deciphering the signals, and actuating prosthetic hand. Researchers are focusing on improving the functionality of prosthetic hand in order to suit the user requirement with the different operating features. The myoelectric control differs in operation to accommodate various external factors. This article reviews the state of the art of myoelectric prosthetic hand, giving description of each control strategy.
\end{abstract}

Keywords: EMG, assistive device, amputee, myoelectric control, electric powered, body powered, bioelectric signal control

\section{Introduction}

Today, the development of science and technology has led to prosthetic devices with promising functional capabilities and esthetic appearance in research domain in favor of commercialization. The design of prosthetic hand is multidisciplinary, compelling knowledge of physiology, anatomy, electrical and electronics, mechanical design, software, and so on, depending on the nature of control. Still, most of the research is in the laboratory and the issue is lack of integration with the technology due to its multidisciplinary nature and the non availability of funds. There have been different types of prosthetic hands ranging from body-powered prosthetic hand to neural interface-based prosthetic hand, which are being manufactured and attempted in the market and for the purpose of research. The choice of prosthetic hand is based on the requirement of the user.

In general, the prosthetic devices could be body powered, pneumatic powered, or electric powered. ${ }^{1}$ The body-powered devices harness energy from muscles to operate the cable through a link. The advantages of body-powered devices are that they are of low cost and are less expensive to repair. However, these devices are not cosmetically appealing and are difficult to operate with body power by some users. The electric-powered prosthetic devices that are operated with battery are desired by most of the users due to their cosmetic appearance. However, these devices are expensive,
Correspondence: P Geethanjali School of Electrical Engineering, Department of Control and Automation, Katpadi-Thiruvalam Road, VIT University, Vellore-632014. Tamil Nadu, India Tel +9l 9944980194 Email pganjali78@hotmail.com 
heavy, and expensive to repair. Nevertheless, there has been a major breakthrough in the operation of electric-powered prosthetic devices. These externally powered devices may be operated from pressure, switch, strain gauge, myoelectric signals (MES), and electroencephalogram signals. There is a possibility of hybrid control strategy to improve the operation of the devices. Regardless of the operation of devices, typically, the prosthetic hands are available with the mechanical design of hooks, prehensors, artificial hands, and special type of terminal devices, depending on the user-specific activity.

Hooks are devices with good durability, less maintenance, low weight, and good gripping capability. The hooks are made out of metals such as aluminum, stainless steel, and titanium. Aluminum has less weight and lower strength, and stainless steel has more weight and strength. Titanium hooks have good strength with less weight. But hooks are not cosmetically appealing. They are used for body-powered control. Prehensors are between hooks and artificial hands. Prehensors are available with/without tension feedback in the market. Similar to hooks, prehensors are not cosmetically appealing and are body powered. The special types of terminal devices made to suit user interest in recreational or sports activities like playing golf, climbing mountain, and so on are also body powered.

Artificial hands are cosmetically pleasing, but functionally inferior to hooks and prehensors. These artificial hands may be controlled using MES, reflecting the intention of the user. Attempts are being made to control the hand through restoration of function of the nerves of arm with targeted muscle reinnervation (TMR) surgery to actuate the hand and through the neural interface. Current state of the art is to control the prosthetic hand using MES with various control schemes to interpret the muscle signals. Figure 1 shows the commercially available body-powered prosthetic hand and myoelectric prosthetic hand from Otto Bock HealthCare GmbH (Duderstadt, Germany). ${ }^{2}$

Attempts are also being made to control the joints of fingers in order to improve the dexterity. The i-limb hand has been developed with the articulation of each finger separately or simultaneously, depending on the capability of the user. Therefore, this review focuses on the state of the art of control of myoelectric-controlled prosthetic hands, giving details of various control strategies and briefing about the mechanical design in commercial and research study.

The myoelectric prosthetic hand is based on electromyographic (EMG) signals generated in skeletal muscles, which reflect the intention of the user. The EMG signals generated by the intention are used to control the prosthetic hand using various deciphering schemes such as proportional control, on-off control, finite state machine, pattern recognition, and postural control. Researchers are attempting to decipher more information from EMG to improve the dexterity of prosthetic hand. On the other hand, some researchers are attempting on EMG signal interfacing techniques to improve the dexterity. Nevertheless, the prosthetic hand control depends on the mode of sensing the signals as well as deciphering the intention from EMG signals. This section presents an overview of literature pertaining to improving myoelectric hand control.

\section{Detection of EMG signals}

The body-powered prosthetic hand does not mimic the natural human hand movement. The user intention-controlled devices mimic the natural human movement. The user intention for the control of hand may be obtained from physiological control signals acquired through sensors. The sensor technology interfaces the human control signals to the artificial hand. Modern prosthetic hands incorporate surface electrode to
A

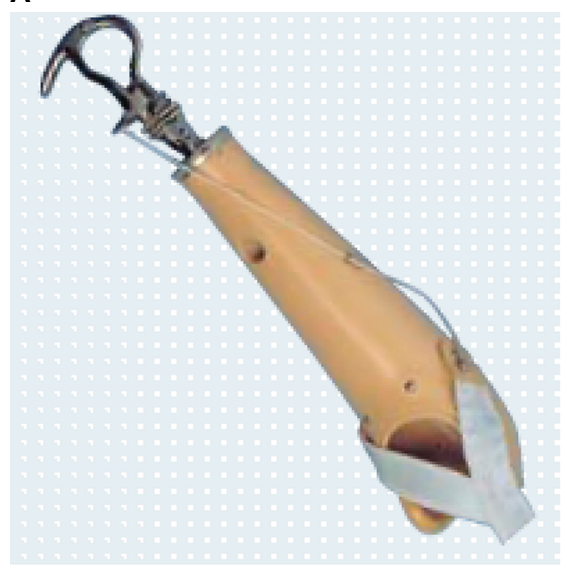

B

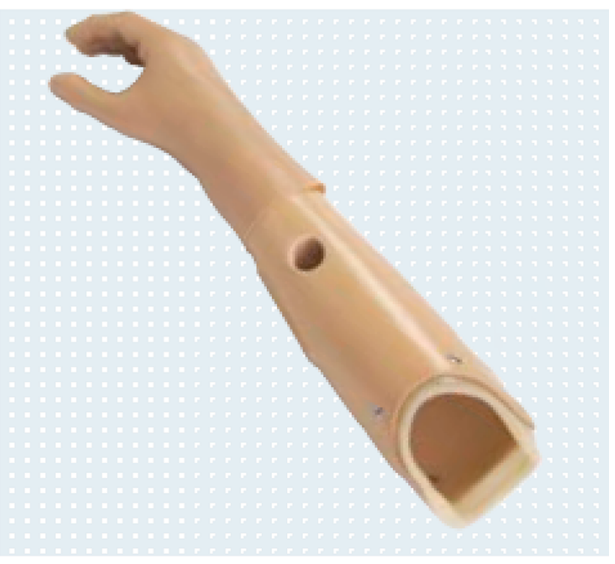


interface artificial hand through myoelectric control signals to human. The surface EMG signals for artificial hand control are sensed from the surface of the skin and are preferred due to their ease of access and the procedure being noninvasive. The dexterity of prosthetic hand is less in surface EMG due to limitation in identifying the locations to acquire signals. Using surface electrodes, it is possible to identify three to four possible locations from the residual limb to acquire signals for sequential control. However, collecting the intramuscular EMG signals $\mathrm{s}^{3-5}$ is an invasive technique and requires surgical skill for using the implantable myoelectric sensor. But the intramuscular EMG signals provide access for collection of EMG signals from multiple locations to offer multiple degrees of control to prosthetic hand. It could be possible to achieve simultaneous control of prosthetic hand with the intramuscular EMG signals using an implantable sensor.

$\mathrm{TMR}^{6}$ surgical procedure has been recently used to rewire the nerves to different muscle sets which can be measured from the surface for the control of artificial hand. The use of TMR is effective for transhumeral amputees, and this technique provides access to utilize user intention.

\section{Myoelectric control schemes}

The EMG signal has been used in prosthetic hand actuation since 1948. ${ }^{7,8}$ Producing commercial prosthetic hand using MES began in 1957 at the Central Prosthetic Research Institute, Moscow to drive stepper motor. ${ }^{9}$ This was later upgraded with permanent magnet DC motor and electromagnetic relays. Later, the myoelectric control strategy had been widely analyzed and a simple on-off control scheme was developed. In this myoelectric control scheme, the amplitude of EMG is used to decode the information in the acquired EMG signals to on/ off state of the motor. The command to actuate the prosthetic device is determined by comparing the amplitude calculated using the root mean square or mean absolute value (MAV) with the preset threshold. A wide variety of control schemes have been developed to translate the information in the EMG and are typically classified based on the nature of control as sequential control and simultaneous control. Most of the control schemes employed in user's prosthetic hand are of sequential control, and research is now being conducted to employ simultaneous control of the hand. In sequential control schemes, the EMG signals are translated using the following schemes: 1) on-off control, 2) proportional control, 3) direct control, 4) finite state machine control, 5) pattern recognition-based control, 6) posture control schemes, and 7) regression control schemes.

The flowchart for implementation of different types of typical myoelectric scheme with the signal processing stages is presented in Figure 2. Furthermore, proportional control is used in combination with direct control, finite state machine, and posture control for effective decoding of information from the MES. The MES signals acquired from the surface of the skin in these schemes are amplified and preprocessed before analog-to-digital conversion. The acquired EMG data are processed to decipher the user intention and communicate with the motor controller in order to actuate the appropriate motor to achieve the user-intended activity. Signal processing of the various modules is described subsequently.

\section{On-off myoelectric control}

The conventional on-off control is appropriate for maximum of two degrees of freedom. In on-off/crisp/binary/bang-bang control, the prosthetic hand is operated with a constant speed in clockwise and counterclockwise directions with a full stop. There are various control schemes for on-off control. The simplest on-off control is based on a threshold of EMG to make a choice of direction of control of the hand. In this control scheme, the hand is operated at a constant speed that is independent of the level of contraction. The simultaneous motion control is possible with motors turned on and off and run at a constant speed. ${ }^{10}$

\section{Proportional myoelectric control}

In proportional control scheme, the voltage applied to the motor is proportional to the contraction level/intensity of EMG signals. ${ }^{11,12}$ This enables fast grasping for gross movement, and the suitability of the control in upper limb is still under study. Researchers have been focusing on simultaneous proportional control recently.

This simultaneous control is against sequential control schemes such as state machine. Other control schemes are used along with proportional control ${ }^{13-16}$ to improve the dexterity in myoelectric control schemes.

\section{Direct myoelectric control}

Direct control ${ }^{17}$ is similar to proportional control and involves independent EMG sites to achieve individual control of finger movements. However, it is difficult to achieve independent control of hand due to crosstalk in EMG signals. This may be possible with intramuscular EMG signals using an implantable myoelectric sensor. ${ }^{9}$

\section{Finite state machine control}

In case of finite state machine control, the postures of the hands are predefined as states and transition among states is also predefined or decoded from the inputs. ${ }^{18,19}$ This is 


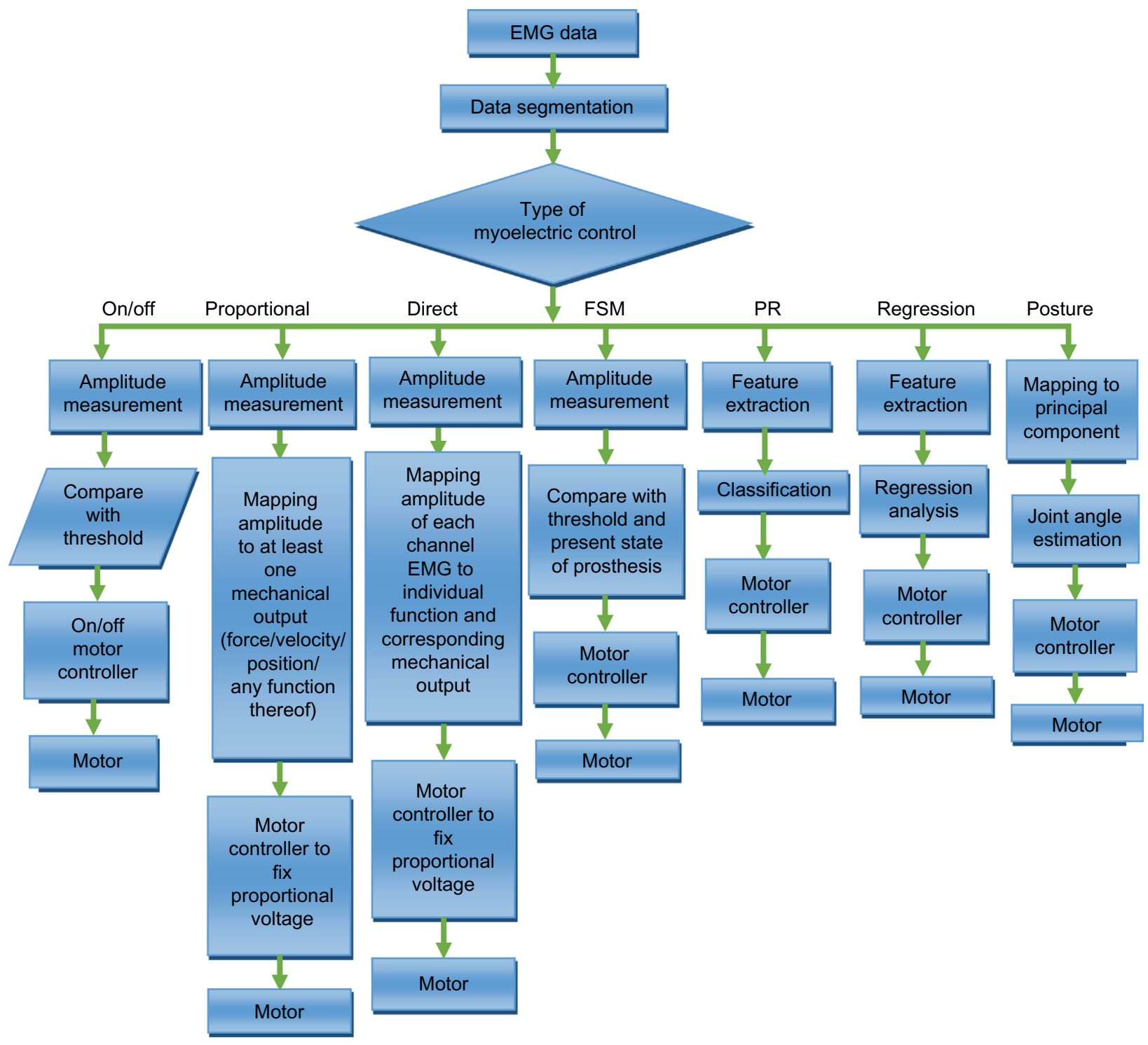

Figure 2 Type of myoelectric control schemes.

Abbreviations: EMG, electromyographic; FSM, finite state machine; PR, pattern recognition.

suitable for a fixed number of postures and may not be suitable for multifunctionality. Furthermore, the state change occurs from the EMG command till the desired posture/ function is selected.

These limitations can be overcome using pattern recognition approach. Many researchers have developed various algorithms for identification of information from the signals using pattern recognition approach.

\section{Pattern recognition-based myoelectric control}

Pattern recognition-based myoelectric control typically consists of feature extraction and feature classification of segmented data in signal processing to command to the motor controller. Some signal processing may include feature reduction or feature selection (FS) between extraction and classification, depending on the number of features. In general, various features are extracted in time, frequency, and time-frequency to identify the information content of the MES.

Pattern-based recognition for myoelectric control involves an effective method of identification of information from the extracted features. Researchers widely used time domain features due to their simplicity. Sardis and Gootee ${ }^{20}$ identified patterns of prespecified motion of the feature space of variance and zero crossing (ZC). Later, Lee and Sardis ${ }^{21}$ used integral absolute value, also known as MAV, along with variance and $\mathrm{ZC}$ for the myoelectric control of the arm. Hudgins et $\mathrm{al}^{22}$ investigated the information content in the transient burst of myoelectric activity using MAV, MAV 
slope, number of ZCs, number of slope sign changes, and waveform length. Because of the stochastic and nonlinear nature of EMG signals, a considerable amount of research has been carried out using autoregressive (AR) models ${ }^{23-28}$ in order to describe feature sets to represent nonstationary nature of EMG signals. Liu et $\mathrm{al}^{28}$ studied the classification accuracy is increasing more rapidly for AR model less than five. The increase in accuracy is low and attains saturation for AR model of five and more. Kang et $\mathrm{al}^{29}$ and Chang et $\mathrm{al}^{30}$ took advantage of the cepstral coefficients of EMG signal as the control command of man-machine interface. Many time domain features have been investigated and compared for their effectiveness in pattern recognition for myoelectric control.

In the frequency domain, the fast Fourier transform $(\mathrm{FFT})^{31-33}$ has been applied to the EMG signals for determining the frequency spectrum of the EMG signal. Farry et $\mathrm{al}^{31}$ used the FFT in teleoperation of prosthetic hand. Sueaseenak et $\mathrm{al}^{33}$ utilized FFT to extract the features from EMG signals of different hand and wrist motions.

More recently, time-frequency and time-space analysis methods have attracted the researchers, but lead to higher dimensional feature vectors and necessitate the use of feature reduction and FS methods to reduce the feature dimension. The short-time Fourier transform, ${ }^{34}$ wavelet transform, ${ }^{35-41}$ and wavelet packet transform ${ }^{36,42,43}$ yield a high dimensional feature vector, and it is essential to employ dimensionality reduction techniques to reduce the burden to the classifier.

Other features such as zero moment, first moment, second moment, and spectral magnitude average from short-time Thompson transform and short-time Fourier transform are used as the features for classification and compared with the performance with temporal features, namely, the integral square, multiple Hamming windows, and multiple trapezoidal windows, for classification of EMG signals. ${ }^{44}$ Feature extraction from EMG using moving approximate entropy, ${ }^{45}$ hidden Markov model (HMM) ${ }^{46} \mathrm{HMM}$-multivariate AR network, ${ }^{47}$ fractal modeling, ${ }^{48,49}$ statistical features about fractal dimensions for classification, ${ }^{50}$ and higher order statistics ${ }^{51}$ are some of the feature vectors that were attempted in pattern recognition.

Due to the multichannel approach used for acquisition of signals, the extracted feature vector dimension can become large. Also, wavelet transforms generate many coefficients to represent time scale features. Thus, dimensionality reduction can be achieved using either FS or feature projection (FP) method. FS requires a search strategy that selects a candidate subset and an objective function that evaluates these candidates. There are many search strategies for FS such as Davies-Bouldin, ${ }^{52-54}$ genetic algorithm, ${ }^{55,56}$ Kohonen's self-organizing map, ${ }^{57}$ particle swarm optimization (PSO),${ }^{58}$ mixture of PSO and the concept of mutual information, ${ }^{59}$ mutual information, ${ }^{60}$ rough set theory, ${ }^{61}$ and multivariate analysis of variances ${ }^{62}$ for selection of features. An important factor that limits the applicability of FS methods to EMG classification problems is the large variance of EMG signals. ${ }^{63}$ Increased number of features can also be reduced by channel selection as proposed by the researchers. The FS algorithms cannot provide powerful performance when the features are dispersed. On the other hand, projection-based methods are more effective than selection-based methods. ${ }^{64}$

Several methods of FP such as principal component analysis (PCA), ${ }^{32,65-67}$ a linear-nonlinear FP composed of PCA and a self-organizing feature map, ${ }^{68}$ simple Fisher linear discriminant analysis (LDA) ${ }^{69}$ LDA-based FP, ${ }^{63}$ uncorrelated LDA, ${ }^{70}$ combination of Fisher LDA, fuzzy logic, and differential evolution, ${ }^{71}$ orthogonal fuzzy neighborhood discriminant analysis, ${ }^{72}$ supervised discretization coupled with $\mathrm{PCA},{ }^{73}$ and individual $\mathrm{PCA}^{74}$ have been attempted to compress a number of features.

The pattern recognition (classification) maps the feature vectors from extracted features into specific classes of motion. Many literature reports highlight the success of neural networks (NNs) and their ability to learn the distinction between different conditions in pattern recognition. The advantage of $\mathrm{NN}$ is its ability to learn linear and nonlinear relationships directly from the data being modeled. As pioneers in developing real-time pattern recognition-based myoelectric control, Kelly et $\mathrm{al}^{75}$ used Hopfield NN to calculate the time series parameter and perceptron network to classify the MES signals. Several researchers used a multilayer perceptron (MLP) NN and various NNs are reported in the literature to classify time domain EMG features for myoelectric control $^{33,48,76-82}$ to classify time domain features. ${ }^{33,48,76-82}$ Wang et $\mathrm{al}^{67}$ applied back-propagation NN with AR coefficients. Zhao et $\mathrm{al}^{78}$ applied Levenberg-Marquardt-based NN with parametric AR model and integral of EMG. Tsuji et al ${ }^{79}$ proposed an NN that combines a common back-propagation NN with recurrent neural filter in order to classify EMG. Other classification techniques are support vector machine, ${ }^{83-85}$ Bayesian classifier, ${ }^{86}$ evidence accumulation, ${ }^{87}$ fuzzy logic, ${ }^{88-90}$ Gaussian mixture model classifier, ${ }^{63}$ Morse code-based classification, ${ }^{91}$ canonical discriminations, ${ }^{92}$ directed acrylic graph support vector machine classification, ${ }^{93,94}$ simple logistic regression, ${ }^{95} k$ nearest neighbor, ${ }^{96} \mathrm{LDA},{ }^{35,83}$ and so on. Furthermore, hybrid classification techniques such as 
HMM-MLP, ${ }^{97}$ HMM-genetic algorithm-MLP, ${ }^{98}$ and neurofuzzy, ${ }^{99,100}$ were attempted to improve the performance of myoelectric control. It is essential to perform signal processing in order to maintain the optimal delay in the controller. ${ }^{101}$ The performance of the pattern recognition method is studied in real time with virtual myoelectric hand control. ${ }^{102}$

But the pattern recognition methods need training to identify the intention of the user, and also, proportional control is deficient. Most of the pattern recognition control strategies are of sequential control. Researchers are now attempting to simultaneously control using pattern recognition method. ${ }^{103,104}$

\section{Posture myoelectric control}

In posture myoelectric control, the EMG signals are mapped to control parameters in the principal component domain. ${ }^{105}$ The principal component domain coordinates are linearly transformed into the joint angle to represent target postures. This posture myoelectric control provides simultaneous myoelectric control of prosthetic hand.

\section{Regression myoelectric control}

Regression strategy is one of the control strategies developed recently to provide simultaneous as well as proportional control. In this control scheme, simultaneous control signals such as various joint angles would be obtained. Researchers attempted using nonnegative matrix factorization, ${ }^{13,14} \mathrm{NN},{ }^{15}$ and other techniques.

\section{Discussion and conclusion}

In this paper, various myoelectric control schemes related to sequential and open loop control in research and not developed as a product have been reviewed. It is essential to develop products based on these schemes. Another milestone in myoelectric control is providing good gripping capability with the joints of digits actuated using motor to mimic human hand gripping. ${ }^{106}$ Furthermore, closed loop control with integration of sensory motor is another thrusting area of research, in addition to simultaneous and proportional control. Researchers are attempting with tactile feedback to close the loop of myoelectric control. ${ }^{107}$ Closed loop control is one of the areas that need to be addressed vehemently. Furthermore, research on implantable myoelectric sensor and TMR is progressing well in developed countries. But these studies should also start in developing countries in order to fill the gap.

Considerable research has been conducted in various parts of the world and it is necessary to measure the viability of myoelectric control strategies from the clinical perspective. In addition to control, other strategies such as mechanical design of hand to improve the dexterity, and providing battery with long life. This also necessitates the integration of experts from various disciplines to make the research clinically viable.

\section{Disclosure}

The author reports no conflicts of interest in this work.

\section{References}

1. Childress DS. Historical aspects of powered limb prosthesis. Clin Prosthet Orthot. 1985;9(1):2-13.

2. Otto Bock HealthCare GmbH; 2002. Available from: http://www. ottobock.com. Duderstadt (DE).

3. Farrell TR, Weir RF. Pilot Comparison of surface vs implanted EMG for multichannel prosthesis control. Proceedings of IEEE 9th International Conference on Rehabilitation Robotics; 2005; Chicago, IL. pp. 277-280.

4. Weir RF, Troyk PR, DeMichele GA, Kerns DA, Schorsch JF, Maas H. Implantable myoelectric sensors (IMESs) for intramuscular electromyogram recording. IEEE Trans Biomed Eng. 2009;56(1):159-171.

5. Al-Ajam Y, Lancashire $\mathrm{H}$, Pendegrass $\mathrm{C}$, et al. The use of a boneanchored device as a hardwired conduit for transmitting EMG signals from implanted muscle electrodes. IEEE Trans Biomed Eng. 2013;60:1654-1659.

6. Kuiken TA, Li G, Lock BA, Lipschutz RD, Stubblefield KA, Englehart K. Targeted muscle reinnervation for real-time myoelectric control of multifunction artificial arms. JAMA. 2009;301(6):619-628.

7. Kobrinskiy AE. Bioelectrical control of prosthetic devices. Her Acad Sci. 1960;30:58-61.

8. Popov B. The bio-electrically controlled prosthesis. J Bone Joint Surg Br. 1965;47:421-424.

9. McKenzie DS. The Russian myoelectric arm. J Bone Joint Surg Br. 1965;47B(3):418-420.

10. Almstrom C. An electronic control system for a prosthetic hand with six degrees of freedom, Tech. Rep. 1:77, Research Laboratory of Medical Electronics, Chalmers University of Technology; 1977.

11. Bottomley AH, Wilson ABK, Nightingale A. Muscle substitutes and myo-electric control. J Br Inst Radio Eng. 1963;26(6):439-448.

12. Sörbye R. Myoelectric controlled hand prostheses in children. Int $J$ Rehab Res. 1977;1:15-25.

13. Muceli S, Farina D. Simultaneous and proportional estimation of hand kinematics from EMG during mirrored movements at multiple degrees-of-freedom. IEEE Trans Neural Syst Rehabil Eng. 2012;20(3):371-378.

14. Muceli S, Jiang N, Farina D. Extracting signals robust to electrode number and shift for online simultaneous and proportional myoelectric control by factorization algorithms. IEEE Trans Neural Syst Rehabil Eng. 2013;22(3):623-633.

15. Nielsen JLG, Holmgaard S, Jiang N, Englehart K, Farina D, Parker P. Enhanced EMG signal processing for simultaneous and proportional myoelectric control. In: Conference Proceedings of IEEE Engineering in Medicine and Biology Society; September 3-6, 2009; Minneapolis.

16. Rehbaum H, Jiang N, Paredes L, Amsuess S, Graimann B, Farina D. Real time simultaneous and proportional control of multiple degrees of freedom from surface EMG: preliminary results on subjects with limb deficiency. Annu Int Conf IEEE Eng Med Biol Soc IEEE. 2012; 2012:1346-1349.

17. Hahne JM, Biessmann F, Jiang N, et al. Linear and nonlinear regression techniques for simultaneous and proportional myoelectric control. IEEE Trans Neural Syst Rehabil Eng. 2014;22(2):269-279.

18. Kyberd PJ, Holland OE, Chappel PH, et al. MARCUS: a two degree of freedom hand prosthesis with hierarchical grip control. IEEE Trans Rehab Eng. 1995;3:70-76. 
19. Cipriani C, Zaccone F, Micera S, Carrozza MC. On the shared control of an EMG-controlled prosthetic hand: analysis of user-prosthesis interaction. IEEE Trans Robot. 2008;24(1):170-184.

20. Sardis GN, Gootee TP. EMG pattern analysis and classification for a prosthetic arm. IEEE Trans Biomed Eng. 1982;BME-29(6):403-412.

21. Lee $S$, Sardis GN. The control of a prosthetic arm by EMG pattern recognition. IEEE Trans Automat Contr. 1984;AC-29(4): 290-302.

22. Hudgins B, Parker P, Scott RN. A new strategy for multifunction myoelectric control. IEEE Trans Biomed Eng. 1993;40(1):82-94.

23. Graupe D, Magnussen J, Beex AA. A microprocessor system for multifunctional control of upper-limb prostheses via myoelectric signal identification. IEEE Trans Automat Contr. 1978;AC-23(4):538-544.

24. Graupe D, Cline WK. Functional separation of EMG signals via ARMA identification methods for prosthesis control purposes. IEEE Trans Syst Man Cybern. 1975;5:252-259.

25. Doerschuk PC, Gustafson DE, Willsky AS. Upper extremity limb function discrimination using EMG signal analysis. IEEE Trans Biomed Eng. 1983;BME-30(1):18-29.

26. Karlik B, Tokhi MO, Alci M. A fuzzy clustering neural network architecture for multifunction upper-limb prosthesis. IEEE Trans Biomed Eng. 2003;50(11):1255-1261.

27. Soares A, Andrade A, Lamounier E, Carrijo R. The development of a virtual myoelectric prosthesis controlled by an EMG pattern recognition system based on neural networks. Intell Inform Syst 2003;21(2):127-141.

28. Liu X, Zhou R, Yang L, Li G. Performance of various EMG features in identifying arm movements for control of multifunctional prostheses. In: Proceedings of IEEE Youth Conference on Information, Computing and Telecommunication; September 20-21, 2009; Beijing, China.

29. Kang WJ, Shiu JR, Cheng CK, Lai JS, Tsao HW, Kuo TS. The application of cepstral coefficients and maximum likelihood method in EMG pattern recognition. IEEE Trans. Biomed Eng. 1995;42(8): 777-785.

30. Chang GC, Kang WJ, Jer-Junn L, et al. Real-time implementation of electromyogram pattern recognition as a control command of manmachine interface. Med Eng Phys. 1996;18(7):529-537.

31. Farry KA, Walker ID, Baramiuk RG. Myoelectric teleoperation of a complex robotic hand. IEEE Trans Robot Autom. 1996;12(5):775-788.

32. Guler NF, Kocer S. Classification of EMG signals using PCA and FFT. J Med Syst. 2005;29(3):29241-29250.

33. Sueaseenak D, Wibirama S, Chanwimalueang T, Pintavirooj C, Sangworasil M. Comparison study of muscular-contraction classification between independent component analysis and artificial neural network. In: Proceedings of International Symposium on Communications and Information Technologies; October 21-23, 2008; Vientiane, Lao.

34. Hannaford B, Lehman S. Short time Fourier analysis of the electromyogram: fast movements and constant contraction. IEEE Trans Biomed Eng. 1986;BME-33(12):1173-1181.

35. Englehart K, Hudgins B. A robust, real-time control scheme for multifunction myoelectric control. IEEE Trans Biomed Eng. 2003; 50(7):848-854.

36. Englehart K, Hudgins B, Parker PA. Time-frequency based classification of the myoelectric signal: static vs dynamic contractions. In: Proceedings of 22nd Annual International Conference of the IEEE EMBS; July 23-28, 2000; Chicago, IL.

37. Jiang MW, Wang RC, Wang JZ, Jin DW. A method of recognizing finger motion using wavelet transform of surface EMG signal. In: Proceedings of 27th Annual International Conference of the IEEE EMBS; January 17-18, 2006; Shanghai, China.

38. Maitrot A, Lucas MF, Doncarli C, Farina D. Signal-dependent wavelets for electromyogram classification. Med Biol Eng Comput. 2005; 43(4):487-492.

39. Zhao J, Jiang L, Cai H, Liu H, Hirzinger G. A novel EMG motion pattern classifier based on wavelet transform and nonlinearity analysis method. In: Proceedings of IEEE International Conference on Robotics and Biomimetics; December 17-20, 2006; Kunming, China.
40. Arvetti M, Gini G, Folgheraiter M. Classification of EMG signals through wavelet analysis and neural networks for controlling active hand prosthesis. In: Proceedings of IEEE 10th International Conference on Rehabilitation Robotics; June 13-15, 2007; Noordwijk, the Netherlands.

41. Kakoty NM, Hazarika SM. Classification of grasp types through wavelet decomposition of EMG signals. In: Proceedings of 2nd International Conference on Biomedical Engineering and Informatics; October 17-19, 2009; Tianjin, China.

42. Li D, Pedrycz W, Pizzi NJ. Fuzzy wavelet packet based feature extraction method and its application to biomedical signal classification. IEEE Trans Biomed Eng. 2005;52(6):1132-1139.

43. Ren X, Huang H, Deng L. MUAP classification based on wavelet packet and fuzzy clustering technique. In: Proceedings of 3rd International Conference on Bioinformatics and Biomedical Engineering; June 11-13, 2009; Beijing, China.

44. Du S, Vuskovic M. Temporal vs. spectral approach to feature extraction from prehensile EMG signals. In: Proceedings of IEEE International Conference on Information Reuse and Integration; November 8-10, 2004; Las Vegas, NV.

45. Ahmad SA, Chappell PH. Surface EMG classification using moving approximate entropy. In: Proceedings of International Conference on Intelligent and Advanced Systems; November 25-28, 2007; Kuala Lumpur, Malaysia.

46. Chan ADC, Englehart KB. Continuous myoelectric control for powered prostheses using hidden Markov models. IEEE Trans Biomed Eng. 2005;52:121-124.

47. Chiang J, Wang ZJ, McKeown MJ. A hidden Markov, multivariate autoregressive (HMM-mAR) network framework for analysis of surface EMG (sEMG) data. IEEE Trans Signal Process. 2008;56(8): 4069-4081.

48. Kirlangic ME, Denizhan Y. Fractal modeling for pattern recognition via artificial neural networks. In: Proceedings of IEEE International Conference on Acoustics, Speech and Signal Processing; June 6-9, 2000; Istanbul, Turkey.

49. Arjunan SP, Kumar DK. Fractal based modeling and analysis of electromyography (EMG) to identify subtle actions. In: Proceedings of 29th Annual International Conference of the IEEE EMBS; August 22-26, 2007; Lyon, France.

50. Xiao H, Zhi-zhong W, Xiao-mei R. Classification of surface EMG signal with fractal dimension. J Zhejiang Univ Sci B. 2005;6B(8):844-848

51. Nazarpour K, Sharafat AR, Firoozabadi SM. A novel feature extraction scheme for myoelectric signals classification using higher order statistics. In: Proceedings of the 2nd International IEEE EMBS Conference on Neural Engineering; March 16-19, 2005; Arlington, VA.

52. Zardoshti-Kermani M, Wheeler BC, Badie K, Hashemi RM. EMG feature evaluation for movement control of upper extremity prosthesis. IEEE Trans Rehabil Eng. 1995;3(4):324-333.

53. Huang H-P, Chen C-Y. Development of myoelectric discrimination systems for a multi-degree prosthetic hand. In: Proceedings of IEEE International Conference on Robotics and Automation; May 10-15, 1999; Detroit, MI.

54. Santa-Cruz R, Riso, Sepulveda F. Optimal selection of time series coefficients for wrist myoelectric control based on intramuscular recordings. In: Proceedings of 23rd Annual International Conference of the IEEE EMBS; 2001; Istanbul, Turkey.

55. Peleg D, Braiman E, Yom-Tov E, Inbar GF. Classification of finger activation for use in a robotic prosthetic arm. IEEE Trans Neural Syst Rehabil Eng. 2002;10(4):290-293.

56. Oskoei MA, Hu H. GA-based feature subset selection for myoelectric classification. In: Proceedings of IEEE International Conference on Robotics and Biomimetics; December 17-20, 2006; Kunming, China.

57. Huang HP, Liu YH, Liu LW, Wong CS. EMG classification for prehensile postures using cascaded architecture of neural networks with selforganizing maps. In: Proceedings of IEEE International Conference on Robotics \& Automation; September 14-19, 2003; Taipei, Taiwan. 
58. Khushaba RN,Al-Jumaily A. Channel and feature selection in multifunction myoelectric control. In: Proceedings of 29th Annual International Conference of the IEEE EMBS; August 23-26, 2007; Lyon, France.

59. Khushaba RN, Al-Ani A, Al-Jumaily A. Swarm intelligence based dimensionality reduction for myoelectric control. In: Proceedings of 3rd International Conference on Intelligent Sensors, Sensor Networks and Information; December 3-6, 2007; Melbourne, QLD, Australia.

60. Yan Z, Wang Z, Xie H. The application of mutual information-based feature selection and fuzzy LS-SVM-based classifier in motion classification. Comput Methods Programs. 2008;90(3):275-284.

61. Yan Z, Wang Z, Xie H. Joint application of rough set-based feature reduction and fuzzy LS-SVM classifier in motion classification. Med Biol Eng Comput. 2008;46(6):519-527.

62. Reischl M, Groll, L, Mikut R. Optimized classification of multiclass problems applied to EMG-control of hand prostheses. In: Proceedings of IEEE International Joint Conference on Neural Network; July 25-29, 2004; Budapest, Hungary.

63. Chu, JU, Moon YJ, Lee SK, Kim SK, Mun MS. A supervised feature-projection-based-real-time EMG pattern recognition for multifunction myoelectric hand control. IEEE Trans Mechatronics. 2007;12(3):282-290.

64. Englehart K. Signal Representation for Classification of the Transient Myoelectric Signal [PhD dissertation]. Canada: University of New Brunswick; 1998.

65. Englehart K, Hudgins B, Parker PA. A wavelet-based continuous classification scheme for multifunction myoelectric control. IEEE Trans Biomed Eng. 2001;48(3):302-310.

66. Smith RJ, Tenore F, Huberdeau D, Etienne-Cummings R, Thakor NV. Continuous decoding of finger position from surface EMG signals for the control of powered prostheses. In: Proceedings of 30th Annual International Conference of the IEEE EMBS; August 20-25, 2008; Vancouver, British Columbia.

67. Wang JZ, Wang RC, Li F, Jiang MW, Jin DW. EMG signal classification for myoelectric teleoperating a dexterous robot hand. In: Proceedings of 27th Annual International conference of the IEEE EMBS; January 17-18, 2006; Shanghai, China.

68. Chu J-U, Moon I, Mun M-S. A real-time EMG pattern recognition system based on linear-nonlinear feature projection for a multifunction myoelectric hand. IEEE Trans Biomed Eng. 2006;53:2232-2239.

69. Oyama T, Mitsukura Y, Karungaru SG, Tsuge S, Fukumi M. Wrist EMG signals identification using neural network. In: Proceedings of 35 th Annual Conference of IEEE Industrial Electronics; November 3-5, 2009; Porto, Portugal.

70. Chan ADC, Green GC. Myoelectric control development toolbox. In: Proceedings of 30th Conference on Canadian Medical and Biological Engineering Society; 2007; Toronto, Canada.

71. Khushaba RN, Al-Ani A, Al-Jumaily A. Orthogonal fuzzy neighborhood discriminant analysis for multifunction myoelectric hand control. IEEE Trans Biomed Eng. 2010;57(6):1410-1419.

72. Khushaba RN, Al-Jumaily A, Al-Ani A. Evolutionary fuzzy discriminant analysis feature projection technique in myoelectric control. Pattern Recogn Lett. 2009;30(7):699-707.

73. Kiatpanichagij K, Afzulpurkar N. Use of supervised discretization with PCA in wavelet packet transformation-based surface electromyogram classification. Biomed Signal Process Control. 2009;4(2):127-138.

74. Hargrove L, Guangline L, Englehart K, Hudgins B. Principal components analysis preprocessing for improved classification accuracies in pattern-recognition-based myoelectric control. IEEE Trans Biomed Eng. 2009;56(5):1407-1414.

75. Kelly MF, Parker PA, Scott RN. The application of neural networks to myoelectric signal analysis: a preliminary study. IEEE Trans Biomed Eng. 1990;37(3):221-230.

76. Tenore F, Ramos A. Towards the control of individual fingers of a prosthetic hand using surface EMG signals. In: Proceedings of 29th Annual International Conference of the IEEE EMBS; August 22-26, 2007; Lyon, France.
77. Tsenov G, Zeghbib AH, Palis F, Shoylev N, Mladenov V. Neural networks for online classification of hand and finger movements using surface EMG signals. In: Proceedings of 8th Seminar on Neural Network Applications in Electrical Engineering; September 25-27, 2006; Belgrade, Serbia.

78. Zhao J, Xie Z, Jiang L, Cai H, Liu H, Hirzinger G. LevenbergMarquardt based neural network control for a five-fingered prosthetic hand. In: Proceedings of IEEE International Conference on Robotics and Automation; April 18-22, 2005; Barcelona, Spain.

79. Tsuji T, Fukuda O, Kaneko M, Koji I. Pattern classification of timeseries EMG signals using neural networks. Int J Adapt Control. 2000;14(8):829-848.

80. Barrero V, Grisales EV, Rosas F, Sanchez C, Leon J. Design and implementation of an intelligent interface for myoelectric controlled prosthesis. In: Proceedings 23rd Annual International Conference of the IEEE EMBS; 2001; Istanbul, Turkey.

81. Jung KK, Kim JW, Lee HK, Chung SB, Eom KH. EMG pattern classification using spectral estimation and neural network. In: Proceedings of Annual Conference on SICE; September 17-20, 2007; Takamatsu, Japan.

82. Duan F, Dai L, Chang W, Chen Z, Zhu C, Li W. sEMG-based identification of hand motion commands using wavelet neural network combined with discrete wavelet transform. IEEE Trans Ind Electron. 2016;63(3):1923-1934.

83. Peng J, Heisterkamp DR, Dai HK. LDA/SVM driven nearest neighbor classification. IEEE Trans Neural Netw. 2003;14(4):940-942.

84. Yang D, Zhao J, Gu Y, Jiang L, Liu H. EMG pattern recognition and grasping force estimation: improvement to the myocontrol of multiDOF prosthetic hands. In: Proceedings of IEEE/RSJ International Conference on Intelligent Robots and Systems; October 10-15, 2009; St. Louis, MO.

85. Lucas MF, Gaufriau A, Pascual S, Doncarli C, Farina D. Multichannel surface EMG classification using support vector machines and signal-based wavelet optimization. Biomed Signal Process Control. 2008;3(2): 169-174.

86. Lei M, Wang ZZ, Cai LY, Zhang HH, Cai H. An EMG classifying method based on Baye's criterion. In: Proceedings of 20th Annual International Conference of the IEEE EMBS; 28 October-1 November, 1998; Hong Kong, Hong Kong.

87. Lee SP, Park SH, Kim JS, Kim IJ. EMG pattern recognition based on evidence accumulation for prosthesis control. In: Proceedings of 18 th Annual International Conference of the IEEE EMBS; 31 October-3 November, 1996; Amsterdam, the Netherlands.

88. Ajiboye AB, Weir RF. A heuristic fuzzy logic approach to EMG pattern recognition for multifunctional prosthesis control. IEEE Trans Neural Syst Rehabil Eng. 2005;13(3):280-291.

89. Boca AD, Park DC. Myoelectric signal recognition using fuzzy clustering and artificial neural networks in real time. In: Proceedings of IEEE International Conference on Neural Networks; 27 June-2 July, 1994; Vol. 5; Orlando City, p. 3098.

90. Micera S, Sabatini AM, Dario P. On automatic identification of upperlimb movements using small-sized training sets of EMG signals. Med Eng Phys. 2000;22(8):527-533.

91. Nilas P, Rani P, Sarkar N. An innovative high-level human-robot interaction for disabled persons. In: Proceedings of IEEE International Conference on Robotics and Automation; 26 April-1 May, 2004; New Orleans, LA.

92. Nagata K, Adno K, Yamada M, Magatani K. A classification method of hand movements using multichannel electrode. In: Proceedings of 27th Annual International Conference of the IEEE EMBS; January 17-18, 2006; Shanghai, China.

93. Zhizeng L, Jian G. Using singular eigenvalues of wavelet coefficient as the input of SVM to recognize motion patterns of the hand. In: Proceedings of International Conference on Neural Networks and Brain; October 13-15, 2005; Beijing, China. 
94. Geethanjali P, Ray KK. A low-cost real-time research platform for EMG pattern recognition-based prosthetic hand. IEEE/ASME Trans Mechatronics. 2015;20(4):1948-1955.

95. Geethanjali P. Comparative study of PCA in classification of multichannel EMG signals. Australas Phys Eng Sci Med. 2015:38:331-343.

96. Kwon, Jangwoo, Lee D, Lee S, Kim N, Hong S. EMG signals recognition for continuous prosthetic arm control purpose. In: Proceedings of IEEE Asia Pacific Conference on Circuits and Systems; November 18-21, 1996; Seoul, Korea.

97. Kwon, Jangwoo, Lee S, Shin C, Jang Y. Signal hybrid HMM-GA-MLP classifier for continuous EMG classification purpose. In: Proceedings of 20th Annual International Conference of the IEEE EMBS; 29 October-1 November, 1998; Hong Kong SAR, China.

98. Khezri M, Jahed M, Sadati N. Neuro-fuzzy surface EMG pattern recognition for multifunctional hand prosthesis control. In: Proceedings of IEEE International Symposium on Industrial Electronics; June 4-7, 2007; Vigo, Spain.

99. Zhang X, Yang Y, Xu X, Zhang M. Wavelet based neuro-fuzzy classification for EMG control. In: Proceedings of International Conference on Communication, Circuits and Systems; 29 June-1 July, 2002; Chengdu, China.

100. Hussein SE, Granat MH. Intention detection using a neuro-fuzzy EMG classifier. IEEE Eng Med Biol Mag. 2002:21(6):123-129.
101. Farrell TR, Weir RF. The optimal controller delay for myoelectric prostheses. IEEE Trans Neural Syst Rehabil Eng. 2007;15: 111-118.

102. Xing K, Yang P, Huang J, Wang Y, Zhu Q. A real-time EMG pattern recognition method for virtual myoelectric hand control. Neurocomputing. 2014;136:345-355.

103. Young A, Smith L, Rouse E, Hargrove L. Classification of simultaneous movements using surface EMG pattern recognition, IEEE Trans Biomed Eng. 2013;60(5):1250-1258.

104. Wurth SM, Hargrove LJ. A real-time comparison between direct control, sequential pattern recognition control and simultaneous pattern recognition control using a Fitts' law style assessment procedure. J Neuroeng Rehabil. 2014;11(91):1-13.

105. Segil JL, Weir RF. Design and validation of a morphing myoelectric hand posture controller based on principal component analysis of human grasping. IEEE Trans Neural Syst Rehabil Eng. 2014;22(2): 249-257.

106. Available from: http://www.touchbionics.com/products/activeprostheses/i-limb-ultra. Access May 01, 2016.

107. Hartmann C, Došen S, Amsuess S, Farina D. Closed-loop control of myoelectric prostheses with electrotactile feedback: influence of stimulation artifact and blanking. IEEE Trans Neural Syst Rehabil Eng. 2015;23(5):807-816.
Medical Devices: Evidence and Research

\section{Publish your work in this journal}

Medical Devices: Evidence and Research is an international, peerreviewed, open access journal that focuses on the evidence, technology, research, and expert opinion supporting the use and application of medical devices in the diagnosis, monitoring, treatment and management of clinical conditions and physiological processes. The identification of novel

\section{Dovepress}

devices and optimal use of existing devices which will lead to improved clinical outcomes and more effective patient management and safety is a key feature. The manuscript management system is completely online and includes a quick and fair peer-review system. Visit http://www. dovepress.com/testimonials.php to read real quotes from authors. 